\title{
Albumin-Binding PSMA Ligands: Implications for Expanding the Therapeutic Window
}

\author{
James M. Kelly ${ }^{1,2}$, Alejandro Amor-Coarasa ${ }^{1,2}$, Shashikanth Ponnala ${ }^{1,2}$, Anastasia Nikolopoulou ${ }^{2,3}$, Clarence Williams, \\ Jr. ${ }^{1,2}$, Stephen G. DiMagno ${ }^{4}$, and John W. Babich ${ }^{1,2,5}$ \\ ${ }^{I}$ Molecular Imaging Innovations Institute (MI3), Department of Radiology, Weill Cornell Medicine, New York, New York; ${ }^{2}$ Division of \\ Radiopharmaceutical Science, Department of Radiology, Weill Cornell Medicine, New York, New York; ${ }^{3}$ Citigroup Biomedical \\ Imaging Center, Weill Cornell Medicine, New York, New York; ${ }^{4}$ College of Pharmacy, University of Illinois-Chicago, Chicago, \\ Illinois; and ${ }^{5}$ Sandra and Edward Meyer Cancer Center, Weill Cornell Medicine, New York, New York
}

Despite significant gains in the treatment of metastatic castrationresistant prostate cancer by radioligands targeting prostate-specific membrane antigen (PSMA), 30\% of patients never respond to therapy. One possible explanation is insufficient dose delivery to the tumor because of suboptimal pharmacokinetics. We have recently described RPS-063, a trifunctional ligand targeting PSMA with high uptake in LNCaP xenograft tumors but also in kidneys. We aimed to use structural modifications to increase the tumor-to-kidney ratio through increased albumin binding and tumor uptake and reduction of kidney activity. Methods: Four structurally related trifunctional PSMA-targeting small molecules were prepared by either varying the albumin-binding group or inserting a polyethylene glycol 8 linker into a common structure. The compounds were ranked by PSMA affinity and albumin affinity and were radiolabeled with ${ }^{68} \mathrm{Ga}$ and

${ }^{177} \mathrm{Lu}$. Tissue kinetics were determined in male BALB/C nu/nu mice bearing LNCaP xenograft tumors. Results: Each of the compounds binds PSMA with a half-maximal inhibitory concentration of no more than $10 \mathrm{nM}$. The albumin-binding group had a minimal effect on PSMA affinity but changed albumin affinity by an order of magnitude. However, the addition of a polyethylene glycol 8 spacer weakened affinity for albumin in each case. Increased affinity for albumin corresponded with delayed blood clearance and modified uptake kinetics in the tumor and kidney. Uptake of ${ }^{177}$ Lu-RPS-072 (34.9 \pm $2.4 \% \mathrm{ID} / \mathrm{g})$ and ${ }^{177} \mathrm{Lu}-\mathrm{RPS}-077(27.4 \pm 0.6 \% \mathrm{ID} / \mathrm{g})$ increased up to $24 \mathrm{~h}$ after injection, and washout by $96 \mathrm{~h}$ was not significant. As a result, the area under the curve (AUC) in the tumor was in the following order: ${ }^{177}$ Lu-RPS-072 > 177Lu-RPS-077 > 177Lu-RPS-063 > 177 Lu-RPS-071. Increased linker length corresponded to more rapid clearance from kidneys. Consequently, the ratio of tumor AUC and kidney AUC was $4.7 \pm 0.3$ for ${ }^{177}$ Lu-RPS-072. Conclusion: The tumor AUC and tumor-to-kidney ratio of ${ }^{177}$ Lu-RPS-072 are significantly enhanced compared with any small molecule investigated in a LNCaP xenograft model to date. In comparison to other PSMAtargeting radioligands that have been evaluated in a PC3-PIP model, activity in kidneys is reduced and activity in tumors compares favorably when the different PSMA expression levels in LNCaP and PC3-PIP cells are considered. RPS-072 therefore exhibits an increased therapeutic index, shows the potential to increase the dose delivered to tumors, and is a highly promising candidate for targeted radioligand therapy.

Received Oct. 1, 2018; revision accepted Nov. 30, 2018.

For correspondence or reprints contact: John W. Babich, Department of Radiology, Weill Cornell Medicine, Belfer Research Building, Room 1600, 413 E. 69th St., New York, NY 10021.

E-mail: job2060@med.cornell.edu

Published online Dec. 14, 2018.

COPYRIGHT (C) 2019 by the Society of Nuclear Medicine and Molecular Imaging.
Key Words: PSMA; radioligand therapy; albumin binding; pharmacokinetics

J Nucl Med 2019; 60:656-663

DOI: 10.2967/jnumed.118.221150

$\mathbf{T}$ reatment of metastatic castration-resistant prostate cancer by radioligand therapy has emerged as a highly promising strategy for reducing tumor burden and increasing overall patient survival. Small molecules labeled with $\beta$-emitting radionuclides that target prostate-specific membrane antigen (PSMA) with high affinity, such as ${ }^{131}$ I-MIP-1095 (1), ${ }^{177}$ Lu-PSMA-617 $(2,3)$, and ${ }^{177} \mathrm{Lu}-$ PSMA (also known as PSMA I\&T) (4), have induced a prostate-specific antigen decline of at least $50 \%$ in approximately $60 \%$ of patients and shown an acceptable safety profile with few severe or irreversible side effects (1-4).

Despite these promising outcomes, more than $30 \%$ of patients never respond to therapy, and the relapse rate remains high (5). Several factors limit the effectiveness of $\beta$-particle radiotherapy, including the modest linear energy transfer of $\beta$-particles ( 6$)$ and poor pharmacokinetics, particularly insufficient dose delivery to the tumor. This limitation is reflected by the recent report that $50 \%$ of injected activity of ${ }^{177}$ Lu-PSMA-617 is excreted within $4 \mathrm{~h}$ of administration and that by $12 \mathrm{~h}$, nearly $70 \%$ of the activity has been excreted (7). It has been reported that there is a significantly positive, even logarithmic, relationship between response rate and dose delivered to the tumor in patients treated with ${ }^{177} \mathrm{Lu}$-DOTATATE (8), and the high dose delivered to prostate cancer metastases is credited for the reported response of these lesions after radioligand therapy with ${ }^{177}$ Lu-PSMA-I\&T (4). A positive correlation between tumor $\mathrm{SUV}_{\max }$ and response rate was reported for patients undergoing therapy with ${ }^{177} \mathrm{Lu}-\mathrm{PSMA}-617$, although this correlation was not found to be statistically significant (9).

In an attempt to increase the dose delivered to tumors, we recently introduced a series of trifunctional ligands with high (halfmaximal inhibitory concentration $\left[\mathrm{IC}_{50}\right]<10 \mathrm{nM}$ ) affinity for PSMA and the ability to bind albumin and chelate trivalent radiometals (10). Among this series, ${ }^{177}$ Lu-RPS-063 showed improved uptake in LNCaP xenograft tumors and minimal uptake in all other organs except the kidneys. The dose integral of this ligand in the tumor during the first $96 \mathrm{~h}$ after injection represented a 4-fold increase over ${ }^{177}$ Lu-PSMA-617. In contrast, ${ }^{177}$ Lu-RPS-067, with an 
extended linker group, cleared rapidly from the blood, as well as from the tumor and the kidneys.

On the basis of our earlier findings, we developed ${ }^{177} \mathrm{Lu}$-RPS072, a molecule that improves even further on the tumor uptake of ${ }^{177} \mathrm{Lu}-\mathrm{RPS}-063$ while dramatically decreasing kidney activity compared with ${ }^{177}$ Lu-RPS-067. Herein, we report its synthesis and evaluation in a preclinical model of prostate cancer.

\section{MATERIALS AND METHODS}

\section{Chemistry}

RPS-063 was synthesized as previously reported (10). RPS-077 was synthesized by the same method from the common precursor, di-tert-butyl (((S)-6-(3-(3-(1- $((S)-10-(((S)-6$-amino-1-(tert-butoxy)-1oxohexan-2-yl)carbamoyl)-2,2-dimethyl-4,12-dioxo-3,15,18,21-tetraoxa5,11-diazatricosan-23-yl)-1H-1,2,3-triazol-4-yl)phenyl)ureido)-1-(tert-butoxy) -1-oxohexan-2-yl)carbamoyl)-L-glutamate (1) (Supplemental Fig. 1; available at http://jnm.snmjournals.org).

RPS-071 and RPS-072 were synthesized from the common precursor di-tert-butyl (((S)-6-(3-(3-(1- $((S)-10-(((S)-33$-amino-29-(tert-butoxycarbonyl)-27-oxo-3,6,9,12,15,18,21,24-octaoxa-28-azatritriacontyl) carbamoyl)-2,2-dimethyl-4,12-dioxo-3,15,18,21-tetraoxa-5,11-diazatricosan-23-yl)-1H-1,2,3-triazol-4-yl)phenyl)ureido)-1-(tert-butoxy)-1oxohexan-2-yl)carbamoyl)-L-glutamate (11) (Supplemental Fig. 2).

A full description of synthetic procedures, including characterization of final compounds, can be found in the supplemental materials.

\section{Radiochemistry}

General Methods. All reagents were purchased from Sigma-Aldrich unless otherwise noted and were of reagent grade. $\mathrm{HCl}$ and $\mathrm{NaOAc}$ were of traceSELECT ( $>99.999 \%$; Sigma-Aldrich) quality. All water used was highly pure $(18 \mathrm{~m} \Omega)$. Analytic high-performance liquid chromatography was performed on a dual-pump Varian Dynamax high-performance liquid chromatography system (Agilent Technologies) fitted with a dual ultraviolet-visible light detector, and radiochemical purity was determined using a $\mathrm{NaI}(\mathrm{Tl})$ flow count detector (Bioscan). Ultraviolet absorption was monitored at 220 and $280 \mathrm{~nm}$. Solvent A was $0.01 \%$ trifluoroacetic acid in $\mathrm{H}_{2} \mathrm{O}$, and solvent B was $0.01 \%$ trifluoroacetic acid in $90 \% \mathrm{v} / \mathrm{v}$ acetonitrile: $\mathrm{H}_{2} \mathrm{O}$. The radiolabeled products were analyzed on a Symmetry C18 column $(5 \mu \mathrm{m}, 2.1$ $\times 50 \mathrm{~mm}, 100 \AA$; Waters) at a flow rate of $2 \mathrm{~mL} / \mathrm{min}$ and a gradient of $0 \% \mathrm{~B}$ to $100 \% \mathrm{~B}$ over $10 \mathrm{~min}$.

Labeling with ${ }^{68} \mathrm{Ga}$. A $1.85-\mathrm{GBq}{ }^{68} \mathrm{Ga} /{ }^{68} \mathrm{Ge}$ generator (Isotope Technologies Garching, $\mathrm{GmbH}$ ) was eluted with $4 \mathrm{~mL}$ of $0.05 \mathrm{M}$ $\mathrm{HCl}$. An aliquot of 1-4 mL containing 155-477 MBq (4.2-12.9 $\mathrm{mCi}$ ) was transferred to a $1.5-\mathrm{mL}$ Eppendorf vial (VWR). Then, $20-40 \mu \mathrm{L}$ of a $1 \mathrm{mg} / \mathrm{mL}$ solution of precursor in dimethyl sulfoxide was added, and the $\mathrm{pH}$ was adjusted to $4.5-5$ by addition of $3 \mathrm{~N}$ NaOAc $\left(20 \mu \mathrm{L}\right.$ per $1 \mathrm{~mL}$ of ${ }^{68} \mathrm{GaCl}_{3}$ solution). The reaction was heated at $95^{\circ} \mathrm{C}$ for $15 \mathrm{~min}$ on an Eppendorf ThermoMixer (Fisher Scientific) before it was taken up in $\mathrm{H}_{2} \mathrm{O}(9 \mathrm{~mL})$ and passed through a preactivated Sep-Pak C18 Plus Light cartridge (Waters). The reaction vial and cartridge were washed with $\mathrm{H}_{2} \mathrm{O}(5 \mathrm{~mL})$, and the cartridge was eluted with $200 \mu \mathrm{L}$ of a $50 \% \mathrm{v} / \mathrm{v} \mathrm{EtOH} / \mathrm{saline}(0.9 \% \mathrm{NaCl}$ solution; VWR) solution, followed by $800 \mu \mathrm{L}$ of saline. The final concentration of each ligand in the reformulated solution was 11-23 $\mu \mathrm{M}$, with a radioactivity concentration of 43.7-206.5 MBq (1.18-5.58 $\mathrm{mCi} / \mathrm{mL}$ ).

Labeling with ${ }^{177} \mathrm{Lu}$. No-carrier-added ${ }^{177} \mathrm{Lu}$ (EndolucinBeta) was purchased from iTG as the chloride salt, with an activity at calibration of 1.5-3.0 GBq. An aliquot containing 0.30-0.72 GBq (8.23-19.58 $\mathrm{mCi}$ ) of the ${ }^{177} \mathrm{Lu}$ stock solution was diluted to $950 \mu \mathrm{L}$ with $0.05 \mathrm{M}$ $\mathrm{HCl}$. To this solution was added $20 \mu \mathrm{g}$ of the precursor as a $1 \mathrm{mg} / \mathrm{mL}$ solution in dimethyl sulfoxide. The $\mathrm{pH}$ was adjusted to $4.5-5$ by addition of $20 \mu \mathrm{L}$ of $3 \mathrm{~N} \mathrm{NaOAc}$, and the buffered solution was heated for $15 \mathrm{~min}$ at $95^{\circ} \mathrm{C}$ on an analog heating block (VWR). After the solution had cooled to room temperature, it was diluted with $\mathrm{H}_{2} \mathrm{O}(9 \mathrm{~mL})$ and passed through a preactivated Sep-Pak C18 Plus Light cartridge (Waters). The cartridge was washed with $\mathrm{H}_{2} \mathrm{O}(5 \mathrm{~mL})$, and the product was eluted with $200 \mu \mathrm{L}$ of EtOH (200 proof, VWR) followed by 800 $\mu \mathrm{L}$ of saline $(0.9 \% \mathrm{NaCl}$ solution; VWR). An aliquot $(50 \mu \mathrm{L})$ was removed from this solution and diluted to $2 \mathrm{~mL}$ with saline. The final concentration of each ligand in the reformulated solution was 0.13$0.23 \mu \mathrm{M}$, with an activity of $3.6-13.4 \mathrm{MBq} / \mathrm{mL}(96-363 \mu \mathrm{Ci} / \mathrm{mL})$.

Cell Culture. The PSMA-expressing human prostate cancer cell line $\mathrm{LNCaP}$ was obtained from the American Type Culture Collection. Cell culture supplies were obtained from Invitrogen unless otherwise noted. LNCaP cells were maintained in RPMI-1640 medium supplemented with $10 \%$ fetal bovine serum (Hyclone), $4 \mathrm{mM}$ L-glutamine, $1 \mathrm{mM}$ sodium pyruvate, $10 \mathrm{mM} \mathrm{N}$-2-hydroxyethylpiperazine- $\mathrm{N}$-2-ethanesulfonic acid, a $2.5 \mathrm{mg} / \mathrm{mL}$ solution of D-glucose, and a $50 \mu \mathrm{g} / \mathrm{mL}$ solution of gentamicin in a humidified incubator at $37^{\circ} \mathrm{C}$ and $5 \% \mathrm{CO}_{2}$. Cells were removed from flasks for passage or for transfer to 12-well assay plates by incubating them with $0.25 \%$ trypsin/ethylenediaminetetraacetic acid.

In Vitro Determination of $I C_{50}$. $\mathrm{IC}_{50}$ values of the nonlabeled, metalfree ligands were determined by screening in a multiconcentration competitive binding assay against ${ }^{99 \mathrm{~m}} \mathrm{Tc}-((7 S, 12 S, 16 S)$-1-(1-(carboxymethyl)$1 H$-imidazol-2-yl)-2-((1-(carboxymethyl)- $1 H$-imidazol-2-yl)methyl)-9, 14-dioxo-2,8,13,15-tetraazaoctadecane-7,12,16,18-tetracarboxylic acid technetium tricarbonyl complex) (99m Tc-MIP-1427), with a dissociation constant $\left(\mathrm{K}_{\mathrm{d}}\right)$ of $0.64 \pm 0.46 \mathrm{nM}$ (11) for binding to PSMA on LNCaP cells, according to previously described methods (12) with small modifications. Briefly, LNCaP cells were plated $72 \mathrm{~h}$ before the experiment to achieve a density of approximately $5 \times 10^{5}$ cells per well (in triplicate) in RPMI1640 medium supplemented with $0.25 \%$ bovine serum albumin. The cells were incubated for $2 \mathrm{~h}$ with $1 \mathrm{nM}{ }^{99 \mathrm{~m} T \mathrm{Tc}-M I P-1427}$ in RPMI-1640 medium containing $0.00125 \% \mathrm{w} / \mathrm{v}$ bovine serum albumin (13) in the presence of $0.001-10,000 \mathrm{nM}$ test compounds. Radioactive incubation medium was then removed by pipette, and the cells were washed twice using $1 \mathrm{~mL}$ of ice-cold phosphate-buffered saline $\times 1$ solution. Cells were harvested from the plates after treatment with $1 \mathrm{~mL}$ of $1 \mathrm{M} \mathrm{NaOH}$ and transferred to tubes for radioactive counting using a 2470 Wizard $^{2}$ automatic $\gamma$-counter (Perkin Elmer). Standard solutions of $10 \%$ injected activity were prepared to enable decay correction. Cell-specific activity was corrected for nonspecific binding of ${ }^{99} \mathrm{~m}$ Tc-MIP-1427. IC $_{50}$ values were determined by fitting the data points to a sigmoidal Hill curve in Origin software.

In Vitro Determination of Albumin Binding. The binding affinity of the ligands for human serum albumin was determined by high-performance affinity chromatography, as previously described, with minor modifications (14). The compounds were labeled with ${ }^{68} \mathrm{Ga}$ as described above and loaded onto a Chiralpak HSA analytic high-performance liquid chromatography column, $100 \times 2 \mathrm{~mm}, 5 \mu \mathrm{m}$ (Daicel Corp.), as a solution in 10\% v/v EtOH/ saline, with a maximum injected mass of $0.8 \mu \mathrm{g}$ and a maximum injected volume of $40 \mu \mathrm{L}$. In addition, retention times for nonradioactive standards of known affinity for HSA (15) were measured. Analyses were performed in triplicate using an isocratic mobile phase of $5 \% \mathrm{v} / \mathrm{v}$ isopropanol $/ 0.067 \mathrm{M}$ phosphate buffer at a constant flow of $0.3 \mathrm{~mL} / \mathrm{min}$. The retention time of each compound was defined as the time of maximum signal in the radio- or ultraviolet chromatogram. The retention times of the radiolabeled compounds were corrected for the offset between the ultraviolet detector and the radiation detector. The apparent $K_{d}$ values were derived from a standard curve defined by the retention times of nonradioactive standards of known affinity for albumin.

Inoculation of Mice with Xenografts. All animal studies were approved by the Institutional Animal Care and Use Committee of Weill Cornell Medicine and were undertaken in accordance with the guidelines 
set forth by the U.S. Public Health Service Policy on Humane Care and Use of Laboratory Animals. Animals were housed under standard conditions in approved facilities with 12-h light/dark cycles. Food and water were provided ad libitum throughout the course of the studies. Male $\mathrm{BALB} / \mathrm{C}$ athymic $n u / n u$ mice were purchased from the Jackson Laboratory. For inoculation in mice, $\mathrm{LNCaP}$ cells were suspended at $4 \times 10^{7}$ cells $/ \mathrm{mL}$ in a 1:1 mixture of phosphate-buffered saline:Matrigel (BD Biosciences). Each mouse was injected in the left flank with $0.25 \mathrm{~mL}$ of the cell suspension. The mice were imaged when the tumors reached approximately $200-400 \mathrm{~mm}^{3}$, and biodistributions were conducted when tumors were in the range of $100-600 \mathrm{~mm}^{3}$.

Small-Animal PET/CT Imaging. Male BALB/C nu/nu mice bearing LNCaP xenograft tumors were injected into the tail vein with 80-100 $\mu \mathrm{L}$ of a $10 \% \mathrm{v} / \mathrm{v} \mathrm{EtOH} /$ saline solution containing $5.2-13.3 \mathrm{MBq}$ $(140-360 \mu \mathrm{Ci})$ and $4 \mu \mathrm{g}(1.8-2.3 \mathrm{nmol})$ of ${ }^{68} \mathrm{Ga}$-labeled ligand. The mice were imaged using small-animal PET/CT (Siemens Inveon) under isoflurane anesthesia $\left(2.5 \%\right.$ in $\left.\mathrm{O}_{2}\right)$ at 1 and $3 \mathrm{~h}$ after injection. Total acquisition time was $30 \mathrm{~min}$, and a CT scan was obtained immediately before the acquisition for anatomic coregistration and attenuation correction. Images were reconstructed using the commercial Inveon software provided by the vendor. Images were corrected for decay and for the total activity injected.

Biodistribution Studies of ${ }^{177}$ Lu-Labeled Ligands in LNCaP Xenograft Mice. LNCaP xenograft tumor-bearing mice $(n=4-5$ per time point per compound) were injected intravenously with a bolus injection of $0.36-1.3 \mathrm{MBq}(9.6-35 \mu \mathrm{Ci})$ and $30-50 \mathrm{ng}(13-23 \mathrm{pmol})$ of each ligand. The mice were killed at 4,24 , and $96 \mathrm{~h}$ after injection. A blood sample was removed, and a biodistribution study was conducted on the following organs (with contents): heart, lungs, liver, small intestine, large intestine, stomach, spleen, pancreas, kidneys, muscle, bone, and tumor. Tissues were weighed and counted on a $2470 \mathrm{Wiz}-$ $\operatorname{ard}^{2}$ automatic $\gamma$-counter. Counts were corrected for decay and for activity injected, and tissue uptake was expressed as percentage injected dose per gram $(\% \mathrm{ID} / \mathrm{g})$ after comparison with a known standard representing $1 \%$ ID. SE measurement was calculated for each data point. Time-activity curves were plotted using Origin software, and the area under the curve (AUC) was determined using the linear trapezoidal rule by implementing the "Integration" function of the software. AUC was expressed as $\% \mathrm{ID} / \mathrm{g}$ per hour. The data for ${ }^{177} \mathrm{Lu}$-RPS-072 were extrapolated to $192 \mathrm{~h}$ after injection by fitting the measurements to an exponential decay function.

Statistics. Statistical analysis of the biodistribution sets was performed using an unpaired $t$ test with GraphPad Prism software. A $P$ value of less than 0.05 was considered significant.

\section{RESULTS}

\section{Chemistry and Radiochemistry}

The precursor ligands were prepared by multistep solutionphase syntheses in greater than $98 \%$ purity, as determined by analytic high-performance liquid chromatography. A full description, including characterization of the final products, can be found

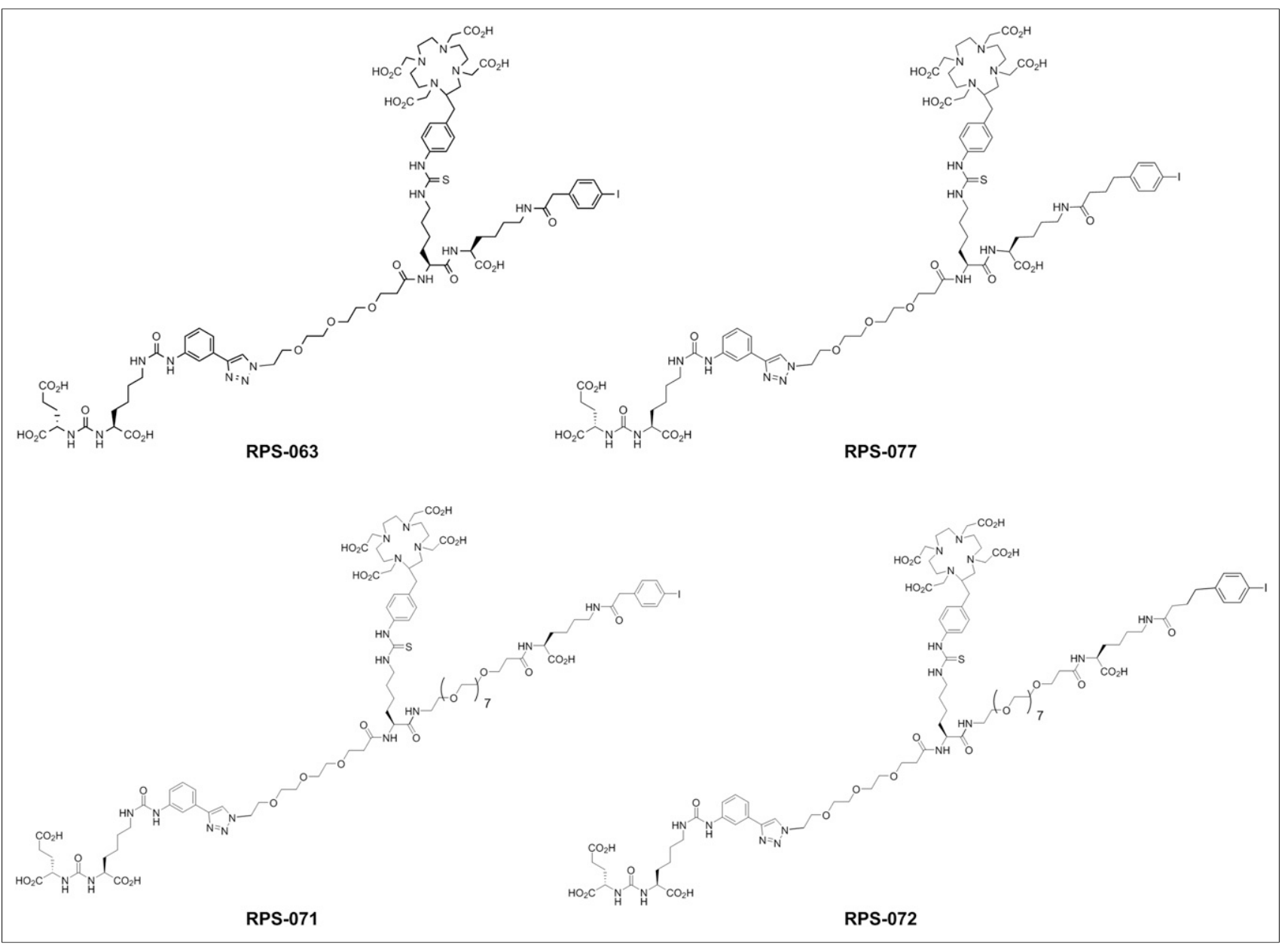

FIGURE 1. Structures of ligands described in this work. RPS-063 was previously reported by Kelly et al. (10). 
in the supplemental materials. All trifunctional ligands studied shared a common structural element incorporating the PSMAtargeting group and DOTA separated by a polyethylene glycol (PEG) 3 linker (Fig. 1). In RPS-063 and RPS-077, the albuminbinding group (ABG) was directly conjugated to this building block via peptide bond, whereas in RPS-071 and RPS-072 the ABG was separated by an additional PEG8 linker.

${ }^{68} \mathrm{Ga}$ labeling was nearly quantitative, although not all radioligand was recovered from the Sep-Pak C18 Plus Light cartridge because of the need to minimize the volume of ethanol in the final solution. Molar activity was greater than $3.96 \mathrm{GBq} / \mu \mathrm{mol}(107$ $\mathrm{mCi} / \mu \mathrm{mol})$, and final radiochemical purity was greater than $99 \%$. The radiochemical yield of the ${ }^{177} \mathrm{Lu}$-labeled products ranged from $67 \%$ to $81 \%(n=4)$ after purification and reformulation, and final radiochemical purity was greater than $98 \%(n=$ 4). A broader range of molar activity, bound by ${ }^{177} \mathrm{Lu}-\mathrm{RPS}-072$ (26.1 GBq/ $\mu \mathrm{mol})$ and ${ }^{177} \mathrm{Lu}-\mathrm{RPS}-071(59.8 \mathrm{GBq} / \mu \mathrm{mol})$, was observed because of differences in starting activity. There was no correlation between starting activity and radiochemical yield.

\section{In Vitro Characterization}

Each trifunctional ligand was found to bind PSMA with high affinity. The $\mathrm{IC}_{50}$ values of RPS-071, RPS-072, and RPS-077 were $10.8 \pm 1.5 \mathrm{nM}, 6.7 \pm 3.7 \mathrm{nM}$, and $1.7 \pm 0.3 \mathrm{nM}$, respectively. The $\mathrm{IC}_{50}$ of RPS-063 was previously reported to be $1.5 \pm 0.3 \mathrm{nM}(10)$. When bovine serum albumin was excluded from the cell medium, nonspecific binding increased considerably, leading to a 5- to 10fold decrease in measured affinity.

By comparing the retention times of the ${ }^{68} \mathrm{Ga}$-labeled ligands on an immobilized HSA column, the relative affinity for albumin was as follows: RPS-077 > RPS-072 > RPS-063 > RPS-071. Affinity was proportional to retention time. ${ }^{68} \mathrm{Ga}$-RPS-071 eluted as a narrow peak after $3.1 \pm 0.2 \mathrm{~min}$ (Supplemental Fig. 4). ${ }^{68} \mathrm{Ga}$-RPS-063 also eluted as a relatively narrow peak, with a retention time of 10.9 $\pm 0.3 \mathrm{~min}$. In contrast, the peak of ${ }^{68} \mathrm{Ga}$-RPS- 072 was broad, and the retention time was $36.1 \pm 1.7 \mathrm{~min} .{ }^{68} \mathrm{Ga}$-RPS-077 did not elute from the column within $80 \mathrm{~min}$. The activity remaining on the column was measured to confirm that the ligand was retained. Retention times were used to derive apparent $\mathrm{K}_{\mathrm{d}}$ values based on a calibration curve generated using standard compounds with known albumin affinity. The $\mathrm{K}_{\mathrm{d}}$ of RPS-063 determined by this method was $1.82 \pm 0.15 \mu \mathrm{M}$. For RPS-071 and RPS-072, the $\mathrm{K}_{\mathrm{d}}$ values were $20.13 \pm 0.79 \mu \mathrm{M}$ and $0.41 \pm 0.01 \mu \mathrm{M}$, respectively. The $\mathrm{K}_{\mathrm{d}}$ of RPS-077 was less than $0.40 \mu \mathrm{M}$, the limit of detection of the assay.

\section{Small-Animal PET/CT Imaging}

At $3 \mathrm{~h}$ after injection, the $4{ }^{68} \mathrm{Ga}$-labeled ligands provided markedly different patterns of distribution, confirming that $4 \mathrm{~h}$ was a reasonable first time point for the biodistributions. The radioligands with higher albumin binding affinity, ${ }^{68} \mathrm{Ga}-\mathrm{RPS}-077$ (Fig. 2A) and ${ }^{68}$ Ga-RPS-072 (Fig. 2B), showed demonstrably greater blood-pool activity. The two with lower albumin affinity, ${ }^{68} \mathrm{Ga}-\mathrm{RPS}-063$ (Fig. 2C) and ${ }^{68} \mathrm{Ga}-\mathrm{RPS}-071$ (Fig. 2D), had substantially less in the blood pool at the same time point. Accumulation of all radioligands was most evident in tumor, kidneys and bladder, although ${ }^{68} \mathrm{Ga}$-RPS-071 and ${ }^{68} \mathrm{Ga}$-RPS-072 had a lower signal in the kidneys than ${ }^{68} \mathrm{Ga}-\mathrm{RPS}-063$ and ${ }^{68} \mathrm{Ga}-\mathrm{RPS}-077 .{ }^{68} \mathrm{Ga}-$ RPS-071 was rapidly excreted via the kidneys.

\section{Biodistribution}

After intravenous administration, the radioligands were predominantly distributed to tumors and kidneys, whereas ${ }^{177} \mathrm{Lu}$-RPS-

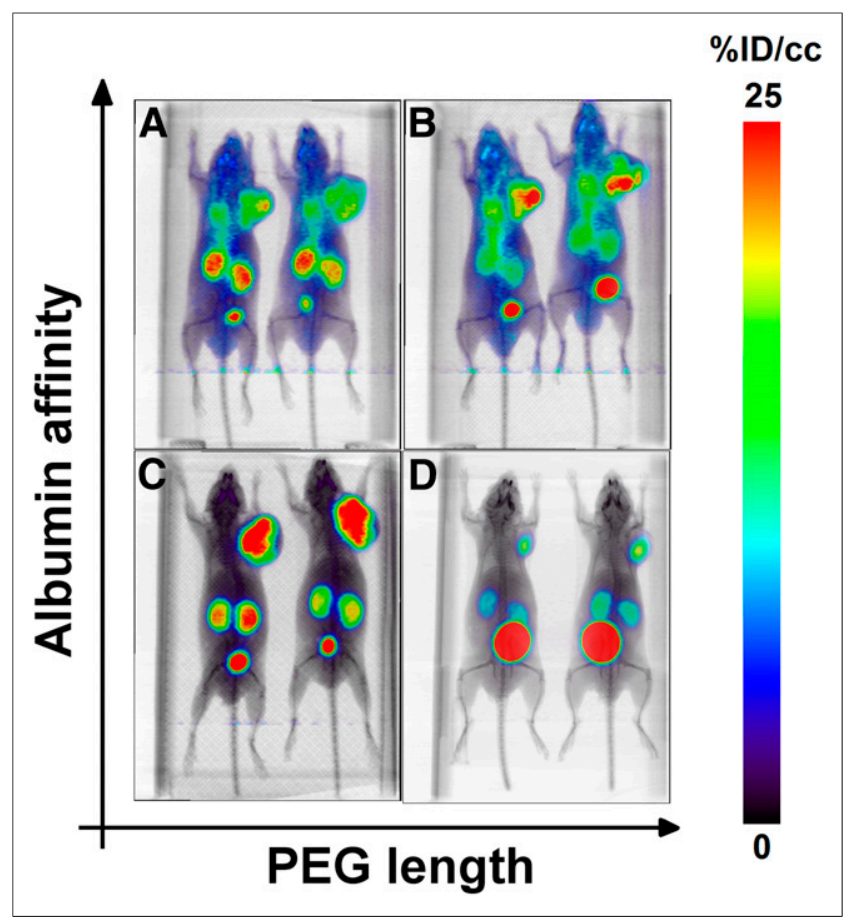

FIGURE 2. Maximum-intensity-projection small-animal PET/CT images of LNCaP xenograft tumor-bearing mice at $3 \mathrm{~h}$ after injection: ${ }^{68} \mathrm{Ga}-\mathrm{RPS}-077$ (A); ${ }^{8} \mathrm{Ga}-\mathrm{RPS}-072$ (B); ${ }^{68} \mathrm{Ga}-\mathrm{RPS}-063$ (C); ${ }^{68} \mathrm{Ga}-\mathrm{RPS}-$ 071 (D). Mice were injected intravenously with 5.2-13.3 MBq (140-360 $\mu \mathrm{Ci}$ ) and imaged for $30 \mathrm{~min}$ under isoflurane. Images are corrected for decay and for activity injected.

072 and ${ }^{177}$ Lu-RPS-077 also persisted in the blood pool (Fig. 3). The biodistribution of ${ }^{177} \mathrm{Lu}-\mathrm{RPS}-063$ was recently published (10) and is recapitulated herein for the purposes of comparison. Activity in the blood correlated with albumin affinity, with ${ }^{177} \mathrm{Lu}$-RPS-077 $(21.9 \pm 0.8 \% \mathrm{ID} / \mathrm{g})\left(\right.$ Fig. 3A) and ${ }^{177} \mathrm{Lu}-\mathrm{RPS}-072(16.0 \pm 1.0 \% \mathrm{ID} / \mathrm{g})$ (Fig. 3B) substantially greater than ${ }^{177} \mathrm{Lu}-\mathrm{RPS}-063(1.1 \pm 0.04 \% \mathrm{ID} / \mathrm{g})$ (Fig. 3C) and ${ }^{177}$ Lu-RPS-071 ( $<0.2 \%$ ID/g) (Fig. 3D) at $4 \mathrm{~h}$ after injection. Clearance from the blood was slowest for ${ }^{177} \mathrm{Lu}-\mathrm{RPS}-$ 077. The activity of ${ }^{177} \mathrm{Lu}-\mathrm{RPS}-072$ and ${ }^{177} \mathrm{Lu}-\mathrm{RPS}-077$ in all other organs decreased with blood clearance, which was more evident in highly vascularized organs such as the kidneys, lungs, and liver.

Uptake of ${ }^{177} \mathrm{Lu}$-RPS-063 in the kidneys was nearly $180 \% \mathrm{ID} / \mathrm{g}$ at $4 \mathrm{~h}$ after injection and still exceeded $50 \% \mathrm{ID} / \mathrm{g}$ at $24 \mathrm{~h}$ (Fig. 3C). In contrast, all other ligands had remarkably low kidney uptake at $4 \mathrm{~h}$ after injection, ranging from $18.2 \pm 1.6 \% \mathrm{ID} / \mathrm{g}\left({ }^{177} \mathrm{Lu}-\mathrm{RPS}-\right.$ $072)$ to $23.6 \pm 0.9 \% \mathrm{ID} / \mathrm{g}$ ( $\left.{ }^{177} \mathrm{Lu}-\mathrm{RPS}-077\right)$. Further, significant clearance $(P<0.008)$ to below $1 \% \mathrm{ID} / \mathrm{g}$ was observed for ${ }^{177} \mathrm{Lu}-$ RPS-071 and ${ }^{177} \mathrm{Lu}$-RPS-072 by $96 \mathrm{~h}$ after injection ${ }^{177} \mathrm{Lu}-\mathrm{RPS}-$ 077, however, continued to accumulate in the kidneys for as long as $24 \mathrm{~h}$ after injection, reaching a maximum of $29.6 \pm 1.8 \% \mathrm{ID} / \mathrm{g}$ before decreasing to $13.0 \pm 1.8 \% \mathrm{ID} / \mathrm{g}$ at $96 \mathrm{~h}$.

As ${ }^{177}$ Lu-RPS-072 activity cleared from the blood, the only tissue in which activity increased was the tumor. The initial tumor uptake of $20.7 \pm 1.9 \% \mathrm{ID} / \mathrm{g}$ at $4 \mathrm{~h}$ after injection increased to 34.9 $\pm 2.4 \% \mathrm{ID} / \mathrm{g}$ at $24 \mathrm{~h}$ after injection, and the magnitude of this change was nearly identical to the change in blood activity over the same period. Subsequent clearance from the tumor was not significant $(P=0.09)$, with $24.8 \pm 4.4 \% \mathrm{ID} / \mathrm{g}$ remaining at $96 \mathrm{~h}$ 


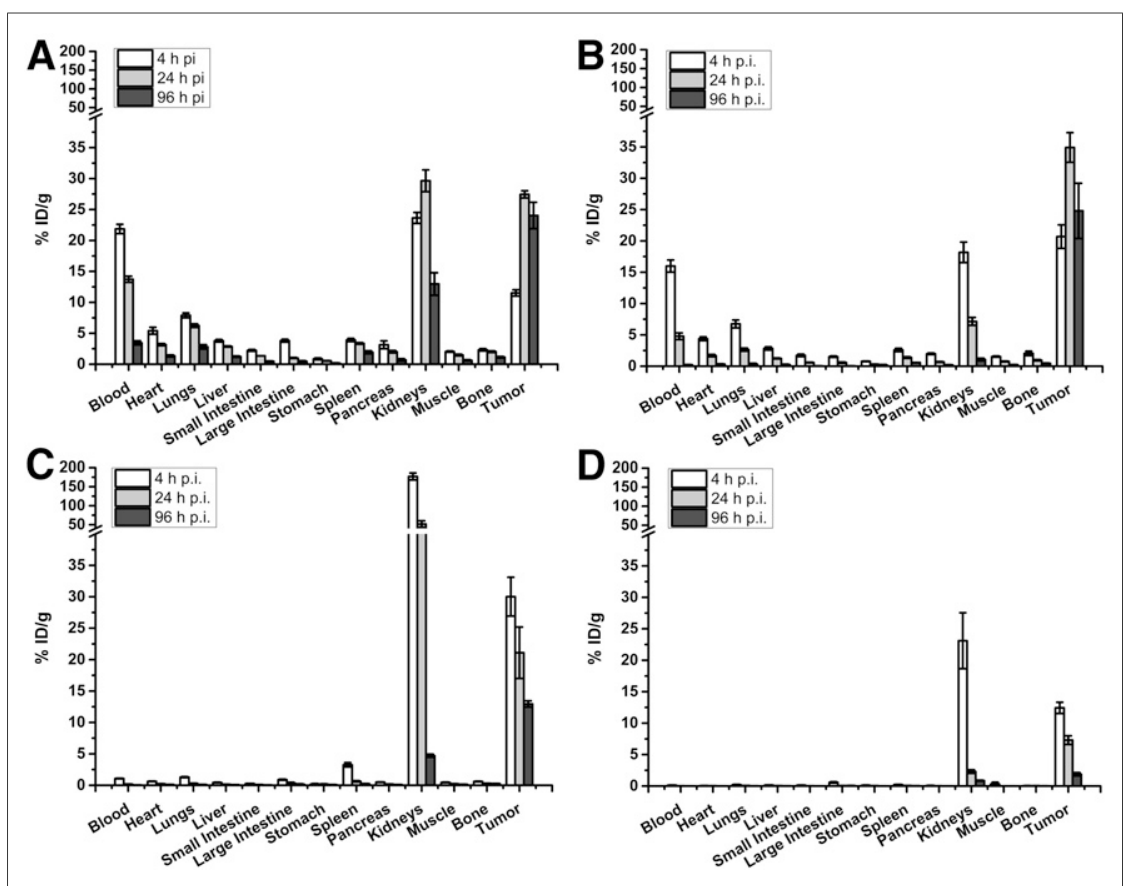

FIGURE 3. Biodistribution of ${ }^{177}$ Lu-RPS-077 (A), ${ }^{177}$ Lu-RPS-072 (B), ${ }^{177}$ Lu-RPS-063 (C), and ${ }^{177} \mathrm{Lu}-\mathrm{RPS}-071$ (D) in male BALB/C athymic nu/nu mice bearing LNCaP xenograft tumors. Panel $C$ was adapted from a previously published figure (10). Mice $(n=4-5 /$ time point) were injected intravenously and sacrificed at 4,24 , or $96 \mathrm{~h}$ after injection (p.i.).

after injection. In concert with clearance from kidneys, the persistent tumor uptake resulted in a tumor-to-kidney ratio for ${ }^{177} \mathrm{Lu}-$ RPS-072 of $22.5 \pm 5.7$ at $96 \mathrm{~h}$ after injection. A similar increase in tumor activity from $4 \mathrm{~h}(11.5 \pm 0.5 \% \mathrm{ID} / \mathrm{g})$ to $24 \mathrm{~h}$ after injection $(27.4 \pm 0.6 \% \mathrm{ID} / \mathrm{g})$ was observed for ${ }^{177} \mathrm{Lu}-\mathrm{RPS}-077$, and uptake $(24.0 \pm 2.1 \% \mathrm{ID} / \mathrm{g})$ remained high up to $96 \mathrm{~h}(P=$ 0.17 ), as noted with ${ }^{177} \mathrm{Lu}-\mathrm{RPS}-072$. However, because of slower kidney clearance, the tumor-to-kidney ratio of ${ }^{177} \mathrm{Lu}$-RPS-077 was only $1.85 \pm 0.30$ at $96 \mathrm{~h}$ after injection.

Uptake of ${ }^{177}$ Lu-RPS-063 and ${ }^{177}$ Lu-RPS-071 in tumors followed different kinetics, with maximum activity measured at $4 \mathrm{~h}$ after injection $(30.0 \pm 3.1 \% \mathrm{ID} / \mathrm{g}$ and $12.4 \pm 0.9 \% \mathrm{ID} / \mathrm{g}$, respectively) and subsequent washout proving to be significant by $24 \mathrm{~h}$ $\left({ }^{177}\right.$ Lu-RPS-071; $\left.P<0.004\right)$ or $96 \mathrm{~h}\left({ }^{177}\right.$ Lu-RPS-063; $P<$ 0.0006). Because of high uptake in the kidneys at early time points, the tumor-to-kidney ratio for ${ }^{177} \mathrm{Lu}$-RPS-063 was less than 1 until $96 \mathrm{~h}$ after injection. At $24 \mathrm{~h}$ the tumor-to-kidney ratio of ${ }^{177} \mathrm{Lu}$-RPS-077 was $3.17 \pm 0.52$, but the significant tumor washout caused the ratio to fall to $2.18 \pm 0.35$ at $96 \mathrm{~h}$ after injection.

Generation of the time-activity curves for the tumor, the blood, and the kidneys was based on the non-decay-corrected biodistribution data (Fig. 4). The AUC was determined for each compound in each organ over the 96-h interval (Table 1). ${ }^{177} \mathrm{Lu}-\mathrm{RPS}-072$ showed the greatest AUC in the tumor $(2747 \pm 145 \% \mathrm{ID} / \mathrm{g}$ per hour), followed by ${ }^{177} \mathrm{Lu}-\mathrm{RPS}-077$ (2266 $\pm 306 \%$ ID/g per hour). The half-life of ${ }^{177} \mathrm{Lu}$-RPS-072 in the tumor was estimated to be $39 \mathrm{~h}$, and the half-life of ${ }^{177} \mathrm{Lu}-\mathrm{RPS}-063$ to be $23 \mathrm{~h}$. In parallel with the findings in the tumor, 2 distinct groups were evident in the blood time-activity curves, as ${ }^{177}$ Lu-RPS-077 $(1,018 \pm$ $42 \% \mathrm{ID} / \mathrm{g}$ per hour) and ${ }^{177} \mathrm{Lu}-\mathrm{RPS}-072(420 \pm 16 \% \mathrm{ID} / \mathrm{g}$ per hour) far exceeded the activity of ${ }^{177} \mathrm{Lu}-\mathrm{RPS}-063$ and ${ }^{177} \mathrm{Lu}-$
RPS-071. The AUC of ${ }^{177}$ Lu-RPS-063 $(4,677 \pm 16 \% \mathrm{ID} / \mathrm{g}$ per hour $)$ in the kidneys was 2-fold greater than ${ }^{177} \mathrm{Lu}$-RPS077 and 10-fold greater than ${ }^{177} \mathrm{Lu}$-RPS-071 and ${ }^{177} \mathrm{Lu}-\mathrm{RPS}-072$. As a result, the tumorto-kidney ratio of ${ }^{177} \mathrm{Lu}$-RPS-072 (4.7 \pm $0.3)$ was superior to that of the other radioligands.

\section{DISCUSSION}

Our first-generation trifunctional PSMA ligands suggested that adding an ABG could improve tumor uptake but that the PEG linker length directly affected albumin affinity and synergistically influenced pharmacokinetics, including clearance from blood and kidneys (10). We therefore hypothesized that modification of the ABG or the PEG linker length could combine the gains in tumor uptake associated with longer blood circulation with the reduction in kidney uptake associated with extended PEG linkers. RPS-063 was selected as the parent structure because of high uptake in LNCaP xenograft tumors and the high yield and purity with which it could be synthesized. A 4-(4-iodophenyl)butanoic acid group was chosen as the ABG because it significantly increased the circulation time of small-molecule radioligands $(13,16-18)$. The ABG was conjugated either directly to the parent structure or via a PEG8 linker, chosen to approximate the overall size of RPS-067 (10).

Modification of the ABG in otherwise structurally identical compounds minimally affected the PSMA affinity but changed affinity for albumin by at least one order of magnitude. Insertion of the additional linker slightly reduced both PSMA affinity and albumin affinity, possibly because of the increased conformational flexibility of the molecule. The PEG group likely also contributed to variable recovery from the $\mathrm{C} 18$ cartridge during purification of the radiolabeled ligands through interactions between the oxophilic silica and the oxygen atoms of the PEG groups.

The AUCs of the 4 radioligands in the blood correlated with the relative albumin affinity determined in vitro, confirming that albumin binding is a significant driving force behind the kinetics of blood clearance. The kinetics of tumor uptake and washout also corresponded to albumin binding affinity. Activity of the 2 weaker binding ligands, ${ }^{177} \mathrm{Lu}-\mathrm{RPS}-063$ and ${ }^{177} \mathrm{Lu}-\mathrm{RPS}-071$, decreased by more than $50 \%$ in tumors between $4 \mathrm{~h}$ and $96 \mathrm{~h}$ after injection. In contrast, 4-(4-iodophenyl)butanoic acid-containing ${ }^{177} \mathrm{Lu}$-RPS-072 and ${ }^{177} \mathrm{Lu}$-RPS-077 reached a maximum uptake at $24 \mathrm{~h}$ after injection, and activity was greater at $96 \mathrm{~h}$ than at $4 \mathrm{~h}$. The maximum tumor uptake of ${ }^{177} \mathrm{Lu}-\mathrm{RPS}-063$ and ${ }^{177}$ Lu-RPS-072 was not statistically different $(P=0.26)$ despite their apparent differences in PSMA affinity in vitro, but the tumor AUC of ${ }^{177}$ Lu-RPS-072 was 1.5-fold higher than that of ${ }^{177} \mathrm{Lu}-\mathrm{RPS}-063$.

The effect of the structural modifications on kidney uptake kinetics was more complex. Within each pair of compounds that varied only by the ABG, the ligands with slower blood clearance, ${ }^{177} \mathrm{Lu}$-RPS-077 and ${ }^{177} \mathrm{Lu}-\mathrm{RPS}-072$, showed an overall 

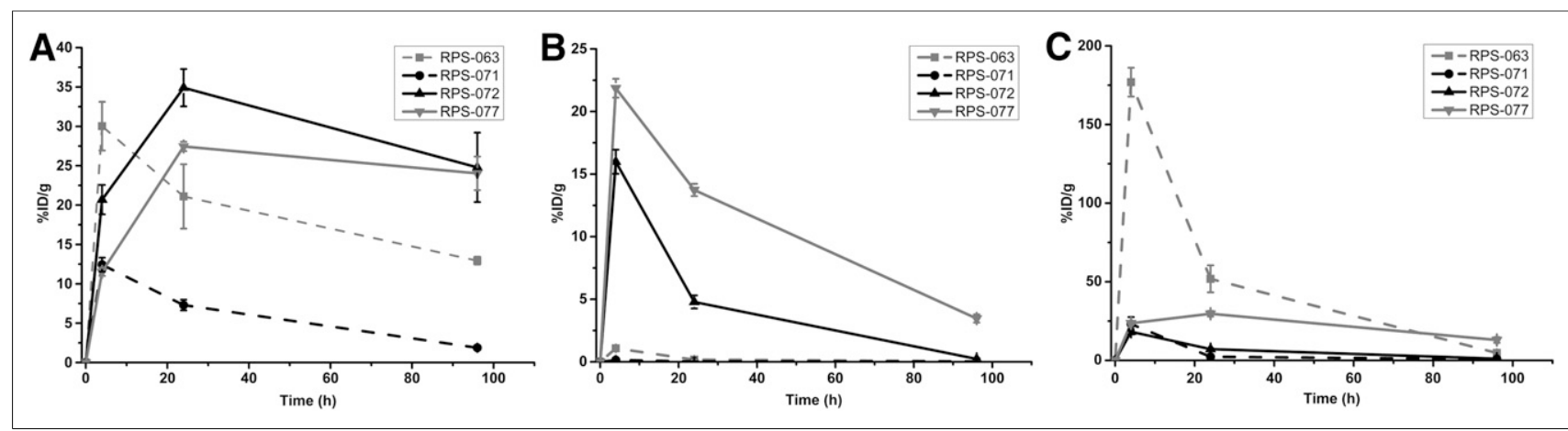

FIGURE 4. Time-activity curves in LNCaP xenograft tumors (A), blood (b), and kidneys (C) of BALB/C nu/nu mice. Curves were plotted using non-decay-corrected biodistribution data. Time-activity curves for ${ }^{177}$ Lu-RPS-063 were derived from previously reported biodistribution values (10).

reduction in kidney activity relative to their 2-(4-iodophenyl)acetic acid-containing analogs, ${ }^{177}$ Lu-RPS-063 and ${ }^{177} \mathrm{Lu}-\mathrm{RPS}-071$, respectively. This reduction was largely due to a lower peak uptake however, because activity at later time points decreased more slowly. A comparison between compounds that differed only by the size of the PEG linker highlighted the dramatic decrease in kidney uptake of the larger radioligands, ${ }^{177}$ Lu-RPS-071 and ${ }^{177} \mathrm{Lu}$-RPS-072, relative to ${ }^{177} \mathrm{Lu}-\mathrm{RPS}-063$ and ${ }^{177} \mathrm{Lu}$-RPS-077, respectively. This reduction is larger than would be predicted by differences in PSMA or albumin affinity alone. Intense accumulation of ${ }^{68} \mathrm{Ga}$-RPS-071 into the bladder at $3 \mathrm{~h}$ after injection suggests that renal activity may peak at an even earlier time point for these larger radioligands. These observations suggest 2 complementary mechanisms for accumulation of activity in the kidney: a component related to renal clearance and a component related to retention in renal proximal tubules. We speculate that enhanced albumin binding slows the rate of renal clearance and that increased linker size reduces retention of the radioligand in the kidney, perhaps by accelerating its clearance before tubular reabsorption. More extensive studies are planned to elucidate the interaction of these ligands and the kidney.

Physiologic uptake of small-molecule PSMA-targeting ligands in kidneys, salivary glands, and lacrimal glands is well established $(1,19,20)$. Dose to kidneys does not appear to be the primary doselimiting toxicity in ${ }^{177} \mathrm{Lu}$-PSMA-617 radioligand therapy. Mildto-moderate xerostomia arising from dose to the salivary glands has been reported after administration of ${ }^{177} \mathrm{Lu}-\mathrm{PSMA}-617$, and salivary gland toxicity is dose-limiting in targeted $\alpha$-therapy with ${ }^{225}$ Ac-PSMA-617 (5). It has proven difficult to assess the impact of PSMA-targeting ligands on the salivary and lacrimal glands in mouse models (13), and so preclinical evaluations of next-generation PSMA ligands have generally used the kidneys as a surrogate for PSMA-expressing tissues. Modification of small-molecule PSMA ligands with albumin-binding groups to increase blood residence time and uptake in tumors has been successfully demonstrated in both LNCaP xenograft models $(10,15)$ and PC3-PIP xenograft models $(13,18,21,22)$, which have been artificially transfected to express high levels of PSMA $(23,24)$. However, these gains in tumor uptake are often short-lived and are achieved in concert with larger increases in kidney activity. It is conceivable that such kidney doses could become relevant in a clinical setting. A phosphoramidate-based PSMA inhibitor, ${ }^{177} \mathrm{Lu}-\mathrm{CTT} 1403$, achieved uptake of greater than $40 \% \mathrm{ID} / \mathrm{g}$ at $48 \mathrm{~h}$ after injection in PC3PIP tumors, but uptake in the kidney at this time point exceeded 50 $\% \mathrm{ID} / \mathrm{g}$, and the tumor-to-kidney ratio was less than 1 for all time points studied (21). The kidney uptake of an Evans blue-glutamate-urea-cysteine conjugate, ${ }^{90}$ Y-DOTA-EB-MCG, decreased relative to the phosphoramidate compound, but activity in the PC3-PIP tumor peaked at only $30 \% \mathrm{ID} / \mathrm{g}$ and was projected to clear completely by $100 \mathrm{~h}$ after injection (22). The tumor-to-kidney ratio of an albumin-binding derivative of PSMA-617, ${ }^{177} \mathrm{Lu}-$ PSMA-Alb-02, improved relative to the phosphoramidate ligands, but although uptake reached $80 \% \mathrm{ID} / \mathrm{g}$ in PC3-PIP tumors, it peaked at $4 \mathrm{~h}$ after injection and decreased rapidly (13). Insertion of a polyglycine linker increased circulation half-life and prolonged tumor loading but also increased kidney uptake. The second-generation ligand ${ }^{177}$ Lu-PSMA-ALB-56 cleared more slowly

TABLE 1

AUCs in Tumor, Blood, and Kidney

\begin{tabular}{lcccc}
\hline \multicolumn{1}{c}{ Parameter } & ${ }^{177}$ Lu-RPS-063 ${ }^{*}$ & ${ }^{177}$ Lu-RPS-071 & ${ }^{177 \text { Lu-RPS-072 }}$ & ${ }^{177 \text { Lu-RPS-077 }}$ \\
\hline Tumor & $1,796 \pm 76$ & $552 \pm 36$ & $2,747 \pm 145$ & $2,266 \pm 306$ \\
Blood & $22 \pm 14$ & $3 \pm 14$ & $420 \pm 16$ & $1,018 \pm 42$ \\
Kidney & $4,676 \pm 14$ & $414 \pm 13$ & $585 \pm 18$ & $2,114 \pm 85$ \\
Tumor-to-blood & $82 \pm 52$ & $184 \pm 859$ & $6.5 \pm 0.4$ & $2.2 \pm 0.3$ \\
Tumor-to-kidney & $0.4 \pm 0.02$ & $1.3 \pm 0.09$ & $4.7 \pm 0.3$ & $1.1 \pm 0.2$
\end{tabular}

*Data derived from previously reported results (10).

Data are \%ID/g per hour. Tumor-to-blood and tumor-to-kidney ratios are derived from corresponding AUCs. 
from the blood, leading to increased PC3-PIP tumor loading with decreased kidney uptake, but tumor washout was extensive after $24 \mathrm{~h}(18)$.

To our knowledge, the tumor-to-kidney AUC ratio of $4.7 \pm 0.3$ achieved by ${ }^{177} \mathrm{Lu}$-RPS-072 over $96 \mathrm{~h}$ is higher than that of any other ligand investigated in a LNCaP xenograft model. Moreover, with nearly $25 \% \mathrm{ID} / \mathrm{g}$ in the tumor and less than $1 \% \mathrm{ID} / \mathrm{g}$ in the kidneys at $96 \mathrm{~h}$ after injection, it is likely that the ratio will continue to grow with time. Extrapolation of the time-activity

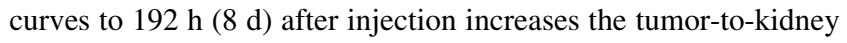
ratio to more than 7.5 and the tumor AUC to nearly 5,000 (\%ID/ g) $\cdot h$ (Supplemental Table 1). Although the AUC of ${ }^{177}$ Lu-PSMAAlb-56 in PC3-PIP tumors was predicted to be nearly twice as high at the same time point, and the tumor-to-kidney dose ratio was estimated to be 11 (18), the artificially high PSMA expression levels in these tumors may favor compounds evaluated in this model relative to those evaluated in an $\mathrm{LNCaP}$ model. This is perhaps most evident in a comparison of the biodistribution of ${ }^{177} \mathrm{Lu}-$ PSMA-617 in each tumor model, for which the AUC(PC3-PIP)/ AUC(LNCaP) ratio is approximately 5:1 (Supplemental Table 1). If this correction factor is applied to ${ }^{177} \mathrm{Lu}$-PSMA-ALB-56, tumor AUC in mice would be reduced to approximately $2,000(\% \mathrm{ID} / \mathrm{g}) \cdot \mathrm{h}$, potentially lower than the dose delivered by ${ }^{177} \mathrm{Lu}$-RPS-072.

Although the relatively long path-length $(670 \mu \mathrm{m})$ and relatively low linear energy transfer of the $\beta$-particle emitted by ${ }^{177} \mathrm{Lu}$ (25) raises the possibility of toxicities associated with the prolonged circulation time of ${ }^{177} \mathrm{Lu}-\mathrm{RPS}-072$, to date only reversible hematologic toxicity has been observed in patients treated with ${ }^{177} \mathrm{Lu}-\mathrm{J} 591$, an antibody in phase II clinical trials that targets the external domain of PSMA (26). Published preclinical data on ${ }^{177} \mathrm{Lu}-\mathrm{J} 591$ are relatively scarce, but the activity levels of ${ }^{111} \mathrm{In}-\mathrm{J} 591$ analog in the blood were found to exceed $4.4 \% \mathrm{ID} / \mathrm{g}$ at $96 \mathrm{~h}$ after injection (27), whereas pharmacokinetic modeling of ${ }^{177} \mathrm{Lu}-\mathrm{J} 591$ in human subjects based on a monoexponential plasma clearance function found a circulation half-life of $39.1 \pm 13.3 \mathrm{~h}(28)$. In comparison, activity of ${ }^{177} \mathrm{Lu}-$ RPS-072 at the same time point in the same xenograft tumor model was less than $1 \% \mathrm{ID} / \mathrm{g}$, and clearance also fit a monoexponential decay function with a half-life of $14.6 \mathrm{~h}$. Consequently, it is likely that the AUC in the blood will be significantly lower for ${ }^{177} \mathrm{Lu}$-RPS-072 than it is for ${ }^{177} \mathrm{Lu}$ J591 and that associated toxicities are therefore unlikely to be dose-limiting.

\section{CONCLUSION}

Through structural modifications at 2 distinct sites within our ligand scaffold, we have demonstrated the ability to manipulate albumin binding and in vivo pharmacokinetics in 2 important ways: tumor uptake and retention, and normal-tissue clearance. Use of a high-affinity albumin-binding group significantly increased blood residence time, leading to increased tumor loading and retention. In conjunction, an extended PEG linker more subtly increased clearance from blood and more dramatically increased clearance from kidneys, without significantly affecting tumor uptake or retention. By fine-tuning pharmacokinetics in this manner, we have developed ${ }^{177} \mathrm{Lu}$-RPS-072 as a highly promising ligand meriting clinical translation for the treatment of prostate cancer by targeted radioligand therapy. The AUC predicts an increase in dose delivered to LNCaP xenograft tumors, and tumor- to-normal-tissue ratios, especially tumor-to-kidney, compare favorably with other ligands of its class.

\section{DISCLOSURE}

James M. Kelly, Alejandro Amor-Coarasa, Shashikanth Ponnala, and John W. Babich are coinventors of the constructs described in this article and hold equity in Noria Therapeutics, Inc. No other potential conflict of interest relevant to this article was reported.

\section{ACKNOWLEDGMENTS}

We thank J. David Warren of the Milstein Chemistry Core Facility at Weill Cornell Medicine for providing equipment used for compound purification and characterization.

\section{REFERENCES}

1. Zechmann CM, Afshar-Oromieh A, Armor T, et al. Radiation dosimetry and first therapy results with a ${ }^{124} \mathrm{I} /{ }^{131} \mathrm{I}$-labeled small molecule (MIP-1095) targeting PSMA for prostate cancer therapy. Eur J Nucl Med Mol Imaging. 2014;41: 1280-1292.

2. Hofman MS, Violet J, Hicks RJ, et al. [ $\left.{ }^{177} \mathrm{Lu}\right]$-PSMA-617 radionuclide treatment in patients with metastatic castration-resistant prostate cancer (LuPSMA trial): a single-centre, single-arm, phase 2 study. Lancet Oncol. 2018;19:825-833.

3. Rahbar K, Bögeman M, Yordanova A, et al. Delayed response after repeated ${ }^{177}$ Lu-PSMA-617 radioligand therapy in patients with metastatic castration resistant prostate cancer. Eur J Nucl Med Mol Imaging. 2018;45:243-246.

4. Baum RP, Kulkarni HR, Schuchardt C, et al. ${ }^{177}$ Lu-labeled prostate-specific membrane antigen radioligand therapy of metastatic castration-resistant prostate cancer: safety and efficacy. J Nucl Med. 2016;57:1006-1013.

5. Kratochwil C, Bruchertseifer F, Giesel FL, et al. ${ }^{225}$ Ac-PSMA-617 for PSMAtargeted $\alpha$-radiation therapy of metastatic castration-resistant prostate cancer. J Nucl Med. 2016;57:1941-1944.

6. Graf F, Fahrer J, Maus S, et al. DNA double strand breaks as predictor of efficacy of the alpha-particle emitter Ac-225 and the electron emitter Lu-177 for somatostatin receptor targeted radiotherapy. PLoS One. 2014;9:e88239.

7. Kurth J, Krause BJ, Schwarzenböck SM, Stegger L, Schäfers M, Rahbar K. External radiation exposure, excretion, and effective half-life in ${ }^{177} \mathrm{Lu}-\mathrm{PSMA}-$ targeted therapies. EJNMMI Res. 2018;8:32.

8. Kulkarni H, Prasad V, Schuchardt C, Zachert C, Baum R. Peptide receptor radionuclide therapy (PRRNT) of neuroendocrine tumors: relationship between tumor dose and molecular response as measured by somatostatin receptor PET/ CT [abstract]. J Nucl Med. 2011;52(suppl 1):301.

9. Ferdinandus J, Eppard E, Gaertner FC, et al. Predictors of response to radioligand therapy of metastatic castrate-resistant prostate cancer with ${ }^{177} \mathrm{Lu}-\mathrm{PSMA}$ 617. J Nucl Med. 2017;58:312-319.

10. Kelly J, Amor-Coarasa A, Ponnala S, et al. Trifunctional PSMA-targeting constructs for prostate cancer with unprecedented localization to LNCaP tumors. Eur J Nucl Med Mol Imaging. 2018;45:1841-1851.

11. Hillier SM, Maresca KP, Lu G, et al. ${ }^{99 \mathrm{~m}}$ Tc-labeled small-molecule inhibitors of prostate-specific membrane antigen for molecular imaging of prostate cancer. J Nucl Med. 2013;54:1369-1376.

12. Kelly J, Amor-Coarasa A, Nikolopoulou A, et al. Synthesis and pre-clinical evaluation of a new class of high-affinity ${ }^{18} \mathrm{~F}$-labeled PSMA ligands for detection of prostate cancer by PET imaging. Eur J Nucl Med Mol Imaging. 2017;44:647-661.

13. Benešová M, Umbricht CA, Schibli R, Müller C. Albumin-binding PSMA ligands: optimization of the tissue distribution profile. Mol Pharm. 2018;15:934946.

14. Hage DS, Anguizola J, Barnaby O, et al. Characterization of drug interactions with serum proteins by using high-performance affinity chromatography. Curr Drug Metab. 2011;12:313-328.

15. Kelly JM, Amor-Coarasa A, Nikolopoulou A, et al. Dual-target binding ligands with modulated pharmacokinetics for endoradiotherapy of prostate cancer. $\mathrm{J} \mathrm{Nucl}$ Med. 2017;58:1442-1449.

16. Müller C, Struthers H, Winiger C, Zhernosekov K, Schibli R. DOTA conjugate with an albumin-binding entity enables the first folic acid-targeted ${ }^{177}$ Lu-radionuclide tumor therapy in mice. J Nucl Med. 2013;54:124-131. 
17. Fischer CR, Groehn V, Reber J, Schibli R, Ametamey SM, Müller C. Improved PET imaging of tumors in mice using a novel ${ }^{18} \mathrm{~F}$-folate conjugate with an albumin-binding entity. Mol Imaging Biol. 2013;15:649-654.

18. Umbricht CA, Benešová M, Schibli R, Müller C. Preclinical development of novel PSMA-targeted radioligands: modulation of albumin-binding properties to improve prostate cancer therapy. Mol Pharm. 2018;15:2297-2306.

19. Afshar-Oromieh A, Malcher A, Eder M, et al. PET imaging with a $\left[{ }^{68} \mathrm{Ga}\right]$ gallium-labelled PSMA ligand for the diagnosis of prostate cancer: biodistribution in humans and first evaluation of tumour lesions. Eur J Nucl Med Mol Imaging. 2013;40:486-495.

20. Afshar-Oromieh A, Hetzheim H, Kratochwil C, et al. The novel theranostic PSMA ligand PSMA-617 in the diagnosis of prostate cancer by PET/CT: biodistribution in humans, radiation dosimetry and first evaluation of tumor lesions. J Nucl Med. 2015;56:1697-1705.

21. Choy CJ, Ling X, Geruntho JJ, et al. ${ }^{177}$ Lu-labeled phosphoramidate-based PSMA inhibitors: the effect of an albumin binder on biodistribution and therapeutic efficacy in prostate tumor-bearing mice. Theranostics. 2017;7:1928-1939.

22. Wang Z, Jacobson O, Tian R, et al. Radioligand therapy of prostate cancer with a long-lasting PSMA targeting agent ${ }^{90}$ Y-DOTA-EB-MCG. Bioconjugate Chem. 2018;29:2309-2315
23. Chang SS, Reuter VE, Heston WDW, Bander NH, Grauer LS, Gaudin PB. Five different anti-prostate-specific membrane antigen (PSMA) antibodies confirm PSMA expression in tumor-associated neovasculature. Cancer Res. 1999;59: 3192-3198.

24. Huang SS, Heston WDW. Should low molecular weight PSMA targeted ligands get bigger and use albumin ligands for PSMA targeting? Theranostics. 2017;7: 1940-1941.

25. Dash A, Pillai MRA, Knapp FF Jr. Production of ${ }^{177} \mathrm{Lu}$ for targeted radionuclide therapy: available options. Nucl Med Mol Imaging. 2015;49:85-107.

26. Tagawa ST, Milowsky MI, Morris M, et al. Phase II study of lutetium-177labeled anti-prostate-specific membrane antigen monoclonal antibody J591 for metastatic castration-resistant prostate cancer. Clin Cancer Res. 2013;19:51825191.

27. Smith-Jones PM, Vallabhajosula S, Navarro V, Bastidas D, Goldsmith SJ, Bander NH. Radiolabeled monoclonal antibodies specific to the extracellular domain of prostate-specific membrane antigen: preclinical studies in nude mice bearing LNCaP human prostate tumor. J Nucl Med. 2003;44:610-617.

28. Vallabhajosula S, Kuji I, Hamacher KA, et al. Pharmacokinetics and biodistribution of ${ }^{111} \mathrm{In}$ - and ${ }^{177} \mathrm{Lu}$-labeled J591 antibody specific for prostate-specific membrane antigen: prediction of ${ }^{90} \mathrm{Y}-\mathrm{J} 591$ radiation dosimetry based on ${ }^{111} \mathrm{In}$ or ${ }^{177}$ Lu? J Nucl Med. 2005;46:634-641. 